\title{
Ventricular assist devices implantation: surgical assessment and technical strategies
}

\author{
Antonio Loforte, Gregorio Gliozzi, Carlo Mariani, Giulio Giovanni Cavalli, Sofia Martin-Suarez, \\ Davide Pacini
}

Cardio-Thorac-Vascular Department, Cardiac Surgery Unit, S. Orsola Hospital, Bologna University, Bologna, Italy

Contributions: (I) Conception and design: A Loforte; (II) Administrative support: D Pacini; (III) Provision of study materials or patients: A Loforte, S Martin-Suarez, D Pacini; (IV) Collection and assembly of data: A Loforte, G Gliozzi, C Mariani, GG Cavalli; (V) Data analysis and interpretation: A Loforte, G Gliozzi, C Mariani, GG Cavalli; (VI) Manuscript writing: All authors; (VII) Final approval of manuscript: All authors.

Correspondence to: Dr. Antonio Loforte, MD, PhD. Cardiothoracic Surgeon, Cardio-Thorac-Vascular Department, Cardiac Surgery Unit, S. Orsola Hospital, ALMA Mater Studiorum University of Bologna, Via Massarenti n.9, 40138 Bologna, Italy. Email: antonioloforte@yahoo.it.

\begin{abstract}
Along with the worldwide increase in continuous left ventricular assist device (LVAD) strategy adoption, more and more patients with demanding anatomical and clinical features are currently referred to heart failure (HF) departments for treatment. Thus surgeons have to deal, technically, with re-entry due to previous cardiac surgery procedures, porcelain aorta, peripheral vascular arterial disease, concomitant valvular or septal disease, biventricular failure. New surgical techniques and surgical tools have been developed to offer acceptable postoperative outcomes to all mechanical circulatory support recipients. Several less invasive and/or thoracotomic approaches for surgery combined with various LVAD inflow and outflow graft alternative anastomotic sites for system placement have been reported and described to solve complex clinical scenarios. Surgical techniques have been upgraded with further technical tips to preserve the native anatomy in case of re-entry for heart transplantation, myocardial recovery or device explant. The current continuous-flow miniaturized and intrapericardial devices provide versatility and technical advantages. However, the surgical planning requires a careful multidisciplinary evaluation which must be driven by a dedicated and well-trained Heart Failure team. Biventricular assist device (BVAD) implantation by adoption of the newer radial pumps might be a challenge. However, the results are encouraging thus remaining a valid option. This paper reviews and summarizes LVAD preoperative assessment and current surgical techniques for implantation.
\end{abstract}

Keywords: Heart failure (HF); ventricular assist devices; surgical strategies; less invasive surgery

Submitted Mar 06, 2020. Accepted for publication Jun 29, 2020.

doi: $10.21037 / \mathrm{cdt}-20-325$

View this article at: http://dx.doi.org/10.21037/cdt-20-325

\section{Introduction}

Over the last decades, there has been a worldwide constant increase in continuous-flow left ventricular assist device (LVAD) adoption and implantation as treatment of endstage heart failure (HF) patients (1-5). Currently, more and more referred patients present complex clinical scenarios encountering surgical re-entry due to previous cardiac procedures, porcelain aorta and peripheral vascular arterial disease, concomitant valvular or septal disease, biventricular failure. Continuous-flow miniaturized and intrapericardial devices along with less invasive surgical (LIS) approaches allow reduced surgical trauma, fitting with most of demanding clinical and anatomical features: optimal inflow and outflow cannulas placement may guarantee better device performances and, consequently, lower rate of pumprelated adverse events and longer pump durability (3). A tailored surgical approach becomes crucial in these potential high risk profiles especially in case of need of anatomy preservation $(4,5)$. In this context, the newer implantable 
Table 1 Preoperative patient assessment.

\begin{tabular}{|c|c|}
\hline Item & Purpose \\
\hline \multirow{4}{*}{ Laboratory } & Coagulation assessment \\
\hline & Heart failure markers \\
\hline & Liver function \\
\hline & Infection and inflammatory markers \\
\hline Chest X-ray & Thoracic aorta assessment, pulmonary emodistribution, pleural effusion \\
\hline Spirometry & Respiratory function assessment \\
\hline Carotid Doppler & Exclude carotid arteries stenosis \\
\hline Transthoracic echocardiography & Assessment of right ventricle (RV) size, geometry, valvulopathies, contractility and function \\
\hline Right heart catheterization & $\begin{array}{l}\text { Assessment of cardiac output (CO), cardiac index }(\mathrm{Cl}) \text {, RV function and reserve (RVSWi), } \\
\text { pulmonary arterial pressure (PAP) and reversibility, pulmonary capillary wedge pressure (PCWP), } \\
\text { right atrial pressure (RAP), pulmonary artery (PA) } \mathrm{SO}_{2}\end{array}$ \\
\hline \multirow[t]{2}{*}{ Computed Tomography (CT) scan } & Thoracic aorta assessment, pulmonary parenchyma assessment \\
\hline & In cases of re-entry (REDO): distance heart/major vessels/sternum assessment \\
\hline
\end{tabular}

centrifugal pumps demonstrated high versatility in different surgical settings.

In the last European Association for Cardio-Thoracic Surgery (EACTS) Expert Consensus document (5), operative recommendations are reported: only standard approach with full median sternotomy, left ventricular (LV) apical inflow and outflow into the ascending aorta, reaches class I recommendation. Other settings should be evaluated and planned case by case, considering also device-specific features, surgeons preference and institutional policies.

In this review, we report all standard and alternative techniques for LVAD implantation even in the case of unviable accesses and anastomosis sites.

\section{Preoperative assessment}

Surgical planning requires a careful multidisciplinary evaluation by a dedicated and well-trained Heart Failure team $(4,5)$ : transthoracic and transesophageal echocardiogram (TEE) provide information on cardiac geometry, ventricular sizes and function, presence of eventual LV apical thrombosis, concomitant valvular disease or septal defects; preoperative right ventricular
(RV) assessment, by echo, right heart catheterization and laboratory, define the risk of postoperative RV failure and may eventually influence the choice of a standard and a less invasive approach. Computed tomography (CT) imaging allows identification of thoracic aorta calcifications and peripheral vascular disease: these anatomical features may impact on cannulation strategy for cardiopulmonary bypass (CPB) or extracorporeal life support (ECLS) installation (central versus peripheral setting) or off-pump implantation need and the choice of inflow cannula and outflow graft anastomotic sites (6). Cannulas position and its relationships with the pump are indeed strongly associated with $\mathrm{LV}$ unloading and early to long-term patient prognosis (7). Moreover, high-resolution lung CT scan and spirometry are necessary to assess the baseline pulmonary function and anatomy (8). Tables 1,2 summarize preoperative and perioperative LVAD potential recipient assessment and management.

\section{Standard approach: full median sternotomy}

Full median sternotomy, LV apex for inflow cannula insertion and ascending aorta for outflow graft placement have been 
Table 2 Perioperative management

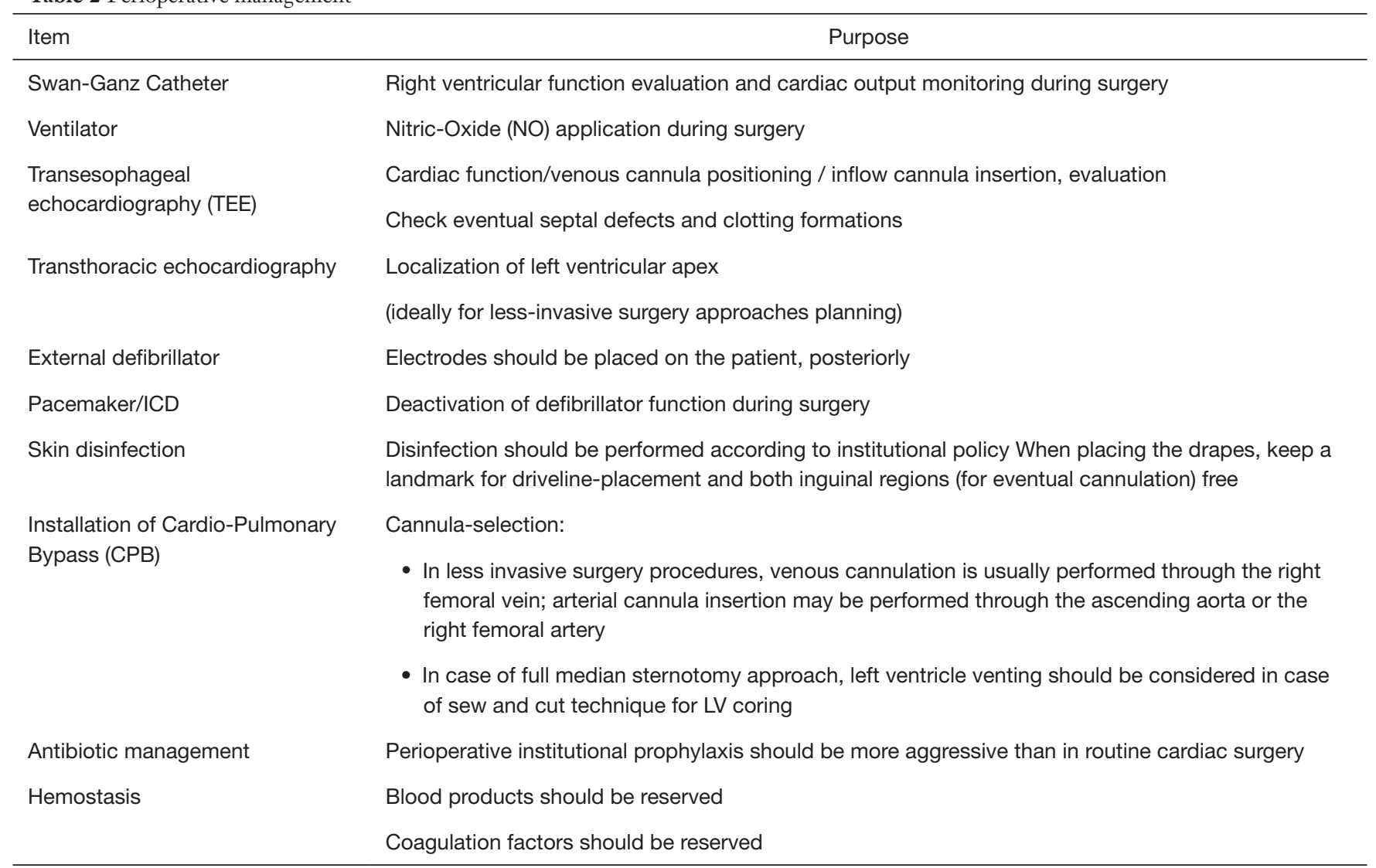
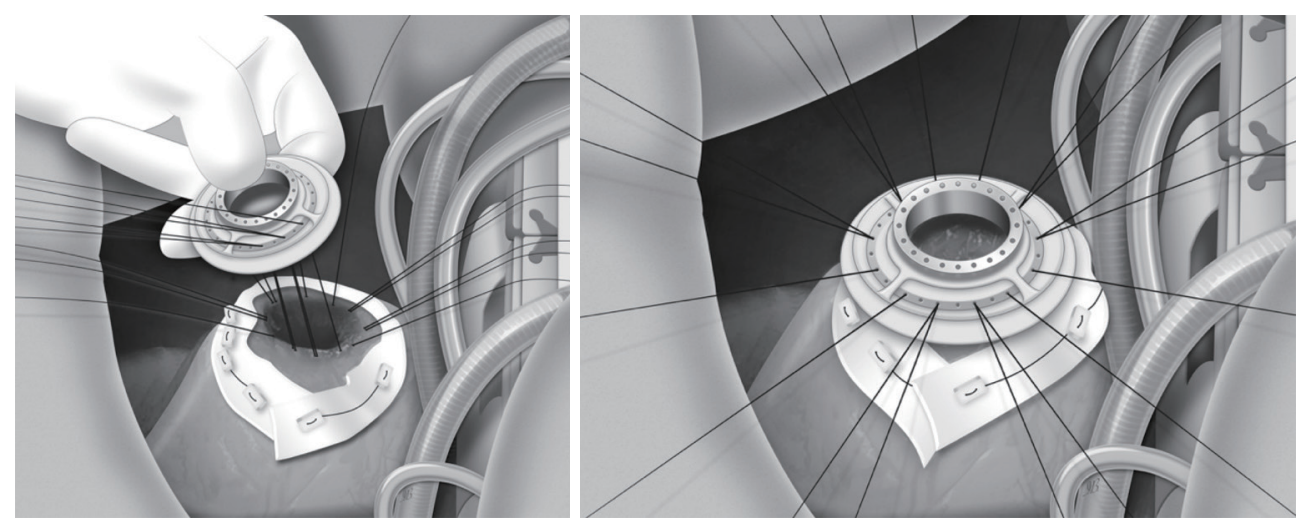

Figure 1 Left ventricular apex sewing ring placement.

the standard approach for decades (Figures 1,2$)(1,5,9)$.

This approach offers a clear view of heart and great vessels. This is essential in concomitant surgeries to be addressed as aortic valve replacement (AVR), mitral and tricuspidal valves repair or replacement, atrial septal defects closure and left atrium appendage exclusion. While AVR may be fully performed by minimally invasive approaches, all the other surgeries may be demanding if performed by LIS.

Limitations of the standard approach are: great surgical trauma with increased risk of postoperative bleeding, full opening of the pericardium with increased risk of RV failure, increased risk of chest instability and wound site 


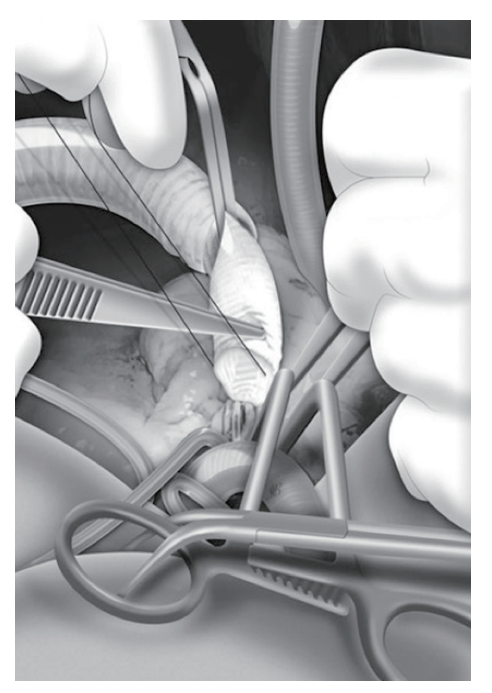

Figure 2 Outflow graft anastomosis on the ascending aorta.

complications $(6,8-28)$.

\section{Sternotomy-sparing approaches: full lateral thoracotomy and less invasive strategies}

International registries reported previous heart surgeries as risk factor for early mortality $(1,10)$. Although re-sternotomy seems not to increase mortality (11), sternotomy-sparing techniques traditionally reduce the risk of postoperative bleeding, infection and impaired wound healing.

Therefore, expert referral centers developed sternotomysparing approaches and identified alternative inflow/outflow anastomotic sites to optimally treat patients with multiple comorbidities, challenging anatomical features and different long-term strategies.

In Table 3 are listed all alternative surgical strategies reported in literature and Figure 3 summarize main features and advantages of each technique $(5,8,12)$.

Initial LIS approaches for LVAD implantation have been adopted for bigger axial devices thus having required even a pre-peritoneal pocket preparation $(9,12-17)$. Hill et al. first reported the idea of a LIS implantation in 3 cases of paracorporeal Thoratec LVAD (Thoratec Corporation, Pleasanton, CA, USA) (13). They suggested the combination of a right mini-thoracotomy and a left subcostal incision. Two successfully underwent heart transplantation. The third one died on LVAD running. Thereafter, other teams by adapting continuous-flow devices, reported optimal results. Gregoric et al. described a LIS approach for HeartMate II LVAD (Thoratec
Table 3 Surgical strategies for left ventricular assist devices (LVADs) implantation

$\begin{array}{ll}\text { Surgical approach } & \text { Full median sternotomy } \\ & \text { Left lateral thoracotomy / subcostal } \\ & \text { Less invasive techniques } \\ & \text { Antero-lateral left thoracotomy + upper } \\ \text { 'J' hemi-sternotomy } \\ \text { - Antero-lateral left thoracotomy + right } \\ \text { parasternal thoracotomy } \\ \text { Left ventricular apex } \\ \text { Left ventricular diaphragmatic wall } \\ \text { Left atrium } \\ \text { Ascending aorta } \\ \text { Descending thoracic aorta } \\ \text { Supra-celiac abdominal aorta } \\ \text { Innominate artery } \\ \text { Axillary artery }\end{array}$

Corporation, Pleasanton, CA, USA) placement (14). They suggested a subcostal incision and access to the pericardium and the LV apex, extraperitoneally, by opening the left pleura. Additionally, a parasternal upper mini-thoracotomy should be performed for ascending aorta anastomosis of the outflow graft. Gregoric et al. performed such an approach successfully in 3 cases $(14,15)$.

Anyanwu et al. applied successfully the sternal-sparing technique with minor modifications (9). Samuels et al. described alternative LIS approaches including a upper hemisternotomy, a left mini-thoracotomy, and a partial upper abdominal pre-peritoneal laparotomy (16). A novel approach has been recently described by the Vienna group to address the case of severe thoracic aorta calcification thus advocating the anastomosys of the outflow graft to the right axillary artery (17).

The first device to be fully placed inside the pericardium by LIS has been the HeartWare HVAD (HeartWare Inc., Framingham, MA, USA; Medtronic) (8,12,18-22). Before surgery it has been recommended to assess each patient in detail, both preoperatively and intraoperatively (Tables 1,2). In order to perform a LIS LVAD implantation, it is necessary a $1^{\text {st }}$ surgical access to the $L V$ apex and a $2^{\text {nd }}$ surgical access to the upper mediastinum (Figure 4) $(8,12,18-22)$.

The LATERAL trial, the first multicenter, prospective, non-randomized, single-arm study, which utilizes data 


\begin{tabular}{|c|c|c|}
\hline \multirow{14}{*}{ Surgical approach } & \multirow{4}{*}{ Full median sternotomy } & Standard approach \\
\hline & & In unstable patients requiring rapid institution of CBP \\
\hline & & In case of high risk of RVF requiring temporary or permanent RVAD \\
\hline & & In case of associated CCP \\
\hline & \multirow{5}{*}{ Left lateral thoracotomy } & In REDO patients with hard re-entry and hostile chest \\
\hline & & Reduced risk of bleeding, impaired wound healing and RVF \\
\hline & & Direct inflow cannula placement \\
\hline & & Alternative outflow graft anastomotic sites \\
\hline & & Anatomy preservation for $\mathrm{Htx}$ or device explant \\
\hline & \multirow{5}{*}{ Less invasive techniques } & In REDO patients with hard re-entry and hostile chest \\
\hline & & Reduced risk of bleeding, impaired wound healing and RVF \\
\hline & & $\begin{array}{l}\text { Ascending aorta easy access for CBP installation, feasibility of outflow graft } \\
\text { anastomosis, feasibility of concomitant AVR }\end{array}$ \\
\hline & & In case of complex LV coring procedure \\
\hline & & Anatomy preservation for $\mathrm{Htx}$ or device explant \\
\hline \multirow{4}{*}{ Inflow graft site } & LV apex & Standard and optimal site \\
\hline & \multirow{2}{*}{ LV diaphragmatic wall } & In case of severe LV dilatation, LV apical aneurysm and calcification, narrow chest \\
\hline & & Anatomy preservation for $\mathrm{Htx}$ or device explant \\
\hline & Left atrium & $\begin{array}{l}\text { Extreme resource in case of restricted LV or unviable LV wall (calcifications and/or } \\
\text { thin wall) }\end{array}$ \\
\hline \multirow{11}{*}{ Outflow graft site } & Ascending aorta & Standard and favourite site \\
\hline & \multirow{3}{*}{ Descending thoracic aorta } & In case of unviable ascending aorta anteriorly and/or in the lateral approach \\
\hline & & In REDO patients with hard re-entry and hostile chest \\
\hline & & Anatomy preservation for $\mathrm{Htx}$ or device explant \\
\hline & \multirow{3}{*}{ Supra-celiac abdominal aorta } & In case of unviable thoracic aorta anteriorly and/or in the lateral approach \\
\hline & & In REDO patients with hard re-entry and hostile chest \\
\hline & & Anatomy preservation for $\mathrm{Htx}$ or device explant \\
\hline & Innominate artery & Suitable with less invasive surgical approaches \\
\hline & \multirow{3}{*}{ Axillary artery } & In case of unviable ascending aorta \\
\hline & & In REDO patients with hard re-entry and hostile chest \\
\hline & & Anatomy preservation for $\mathrm{Htx}$ or device explant \\
\hline
\end{tabular}

Figure 3 Surgical left ventricular assist device (LVAD) settings. AVR, aortic valve replacement or repair; CPB, cardiopulmonary bypass; CCP, concomitant cardiac surgery procedure; Htx, heart transplantation; LV, left ventricular; RVF, right ventricular failure; RVAD, right ventricular assist device. 
from 144 patients enrolled in the Interagency Registry for Mechanically Assisted Circulatory Support (INTERMACS) database at 26 centers in the United States (USA) and Canada, currently validates sternal-sparing approaches for intrapericardial device implantation in a selected bridge to transplantation (BTT) cohort, with similar or even better rates of overall survival and freedom from adverse events than full sternotomy access (23). Many single center experiences reported better survival and lower

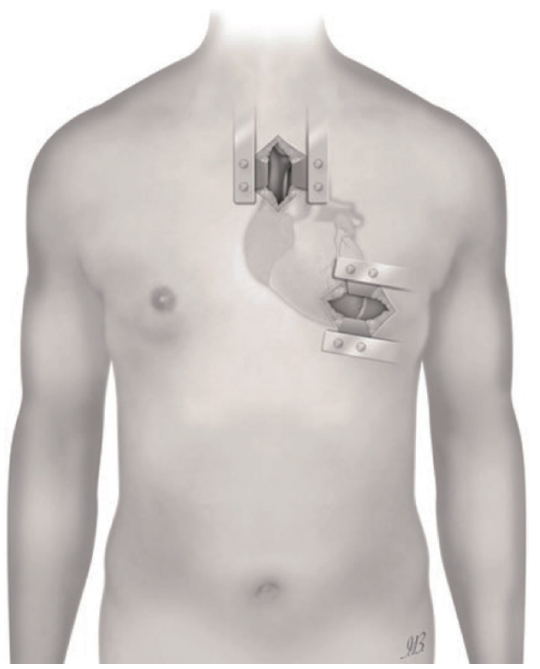

Figure 4 Upper-J ministernotomy and left anterior minithoracotomy for less invasive left ventricular assist device (LVAD) implantation. peri-operative complication rates as well $(8,9,12,18-23)$. The usage of HeartMate 3 (Abbott), even if more 'bulky' resulted to be successful thanks to new tools for minimally invasive surgery provided by the industry (Figure 5) $(24,25)$.

Currently, most of LIS implant techniques consists of an antero-lateral thoracotomy at fifth or sixth intercostal space, over the left ventricle apex, combined with an upper J-shaped mini-sternotomy $(5,8,12,20,21)$. Alternatively, the antero-lateral left thoracotomy may be associated with a right parasternal thoracotomy at second or third intercostal space, according to anatomical relationships and surgical program $(5,8,12,19,22)$. Both strategies, technically modified and implemented by different high-volume centers $(8,12)$, allow exposition of the ascending aorta and epi-aortic vessels for arterial cardio-pulmonary bypass (CPB) cannulation and for the outflow graft anastomosis. Even if the sternal bone is preserved fully, the right-sided thoracotomy may be more painful and the graft anastomosys to the ascending aorta might be more demanding, sometimes $(5,21)$. The upper hemisternotomy may be better suited for redo LVAD cases and concomitant cardiac surgery procedures. This is essential, currently, since a growing number of LVAD recipients already got prior surgical treatments. Moreover, upper hemi-sternotomy potentially enables aortic and tricuspid concomitant surgeries, in expert hands $(5,8,12,21)$.

Although last EACTS Expert Consensus document recommend secured vascular access for $\mathrm{CPB}$, an ideal and complete minimally invasive setting would require an offpump implantation, thus avoiding post-CPB systemic
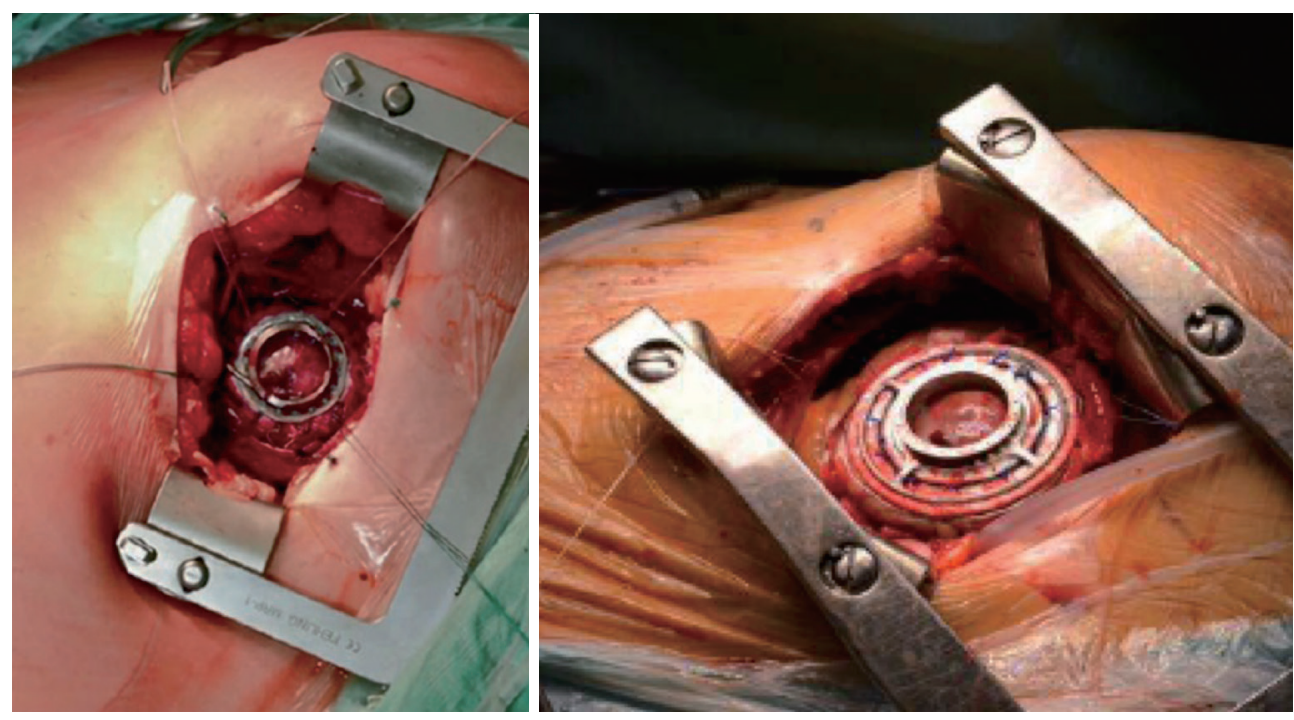

Figure 5 Sewing rings placement in left anterior mini-thoracotomy left ventricular assist device (LVAD) implantation. 
inflammatory disease and improving postoperative course (5,27-29). In case of off-pump procedures, outflow cannula is positioned with a non-fibrillatory technique, pharmacologically-induced bradycardia and a rapid LV apex incision $(5,8,12,18,20)$. CPB is mandatory in case of $\mathrm{LV}$ apical thrombosis or in the presence of a trabecular pattern requiring intraventricular inspection and resection in order to reduce inflow cannula disturbance. Additionally in case of concomitant cardiac surgery during LVAD implantation, CPB remains still necessary. ECLS may be useful in the case unstable patients or crushed patients due cardiogenic shock requiring immediate mechanical circulatory support thus being stabilized first temporarily and then definitively implanted. This may have a significant contribution in terms of less invasive surgery if a full off-pump implantation results to be rescue (5). There is no difference in terms of axial or centrifugal system to be adopted in case of ECLS, $\mathrm{CPB}$ or off-pump strategies. This depends on institutional policy and surgeon's preference.

A single, large, left thoracotomy may be preferred in case of hostile chest wall, unviable ascending aorta and/or previous heart surgery, in particular coronary artery bypass grafting (CABG). The patient is intubated with double lumen tube and placed in right semi-lateral decubitus position; thoracotomy is performed at fifth intercostal space, peripheral CPB is established and outflow graft will be anastomosed to the descending thoracic aorta $(5,12,30)$.

This setting makes surgical cut-down easier, reduces surgical trauma and preserves anatomy (particularly in BTT patients), but on the other hand, offers a poor access to right heart in case of $\mathrm{RV}$ failure requiring mechanical temporary circulatory support $(12,31)$.

Many authors suggest that pericardium integrity (opened only at the level of sewing ring implantation) guarantee an additional, passive stabilization of the RV geometry, avoiding sudden dilatation and reducing postoperative RV failure rate. For this purpose, several perioperative protocols tend to optimize RV unloading through adequate inotropic support, early CPB establishment and low carbon-dioxide mechanical ventilation (18-29,31).

A potential limitation of minimally invasive approach could be its ideal suitability with only few miniaturized and newer devices, such as HeartWare HVAD (Medtronic) and HeartMate 3 (Abbott) or old generation intrapericardial axial-flow pumps (e.g., Jarvik 2000) $(5,8,12)$. Viceversa, left lateral thoracotomy can be applied to a wider range of axial and centrifugal pumps $(5,8,12,18-29)$. Extra-pericardial axial pumps (e.g., HeartMate II) require the creation of a preperitoneal, abdominal pocket for pump housing, through a sub-xiphoid or subcostal incision $(5,12)$.

Although attractive, minimally invasive implantation remains a technically demanding procedure: surgeons must be confident with apical coring and outflow graft alternative anastomotic sites management $(5,8,12)$.

In the future, robotic surgery or trans-catheter LVAD implantation (HVAD prototype) may represent another noteworthy sternotomy sparing approach but, at present, only case-report experiences are reported $(32,33)$.

\section{Inflow cannula placement}

EACTS Expert Consensus document (5) recommends inflow cannula placement into the $\mathrm{LV}$ apex anterior wall (Figure 1), about $2 \mathrm{~cm}$ lateral to the left anterior descending coronary artery, parallel to the interventricular septum towards the apical axis (connecting apex and mitral valve orifice). Angulating the cannula more than $7^{\circ}$ from this axis, as well as pushing cannula too deep into the $\mathrm{LV}$ chamber, negatively influence intracavitary hemodynamics and LV unloading, increasing risk of pump thrombosis (34,35).

Therefore, a careful inflow anastomosis site planning and proper device choice are crucial to obtain the best postoperative outcomes. Small or restricted LV (end diastolic diameter lower than $5.5 \mathrm{~cm}$ ) are at higher risk of postoperative mortality and complications, such as RV failure. In this case, the choice of a centrifugal pump is preferable because it allows to reach a wide range of flows without a significant increase in episodes of suction (36).

Direct access to the apex, as during sternal-sparing approaches, allows a better inflow cannula placement, with low risk of long term device thrombosis and longer pump durability (18-29).

The correct position of sewing ring is identified under trans-esophageal echocardiographic control, through finger or needle localization (5). Sewing ring is fastened to the LV apex with twelve pledgeted prolene sutures, placed deeply into the myocardium; after the X-shaped fullthickness ventriculotomy, the coring is performed and the $\mathrm{LV}$ chamber is inspected and freed from any thrombi or trabeculae crossing the inflow site. The inflow cannula is inserted into the $\mathrm{LV}$, embed in the sewing ring and rotated clockwise or counter-clockwise, according to the outflow anastomotic site (37).

Previous Dor procedure or concomitant LV aneurysm reconstruction represents a challenge because of pericardial adhesions, thin wall, apical calcifications, apex thrombosis 

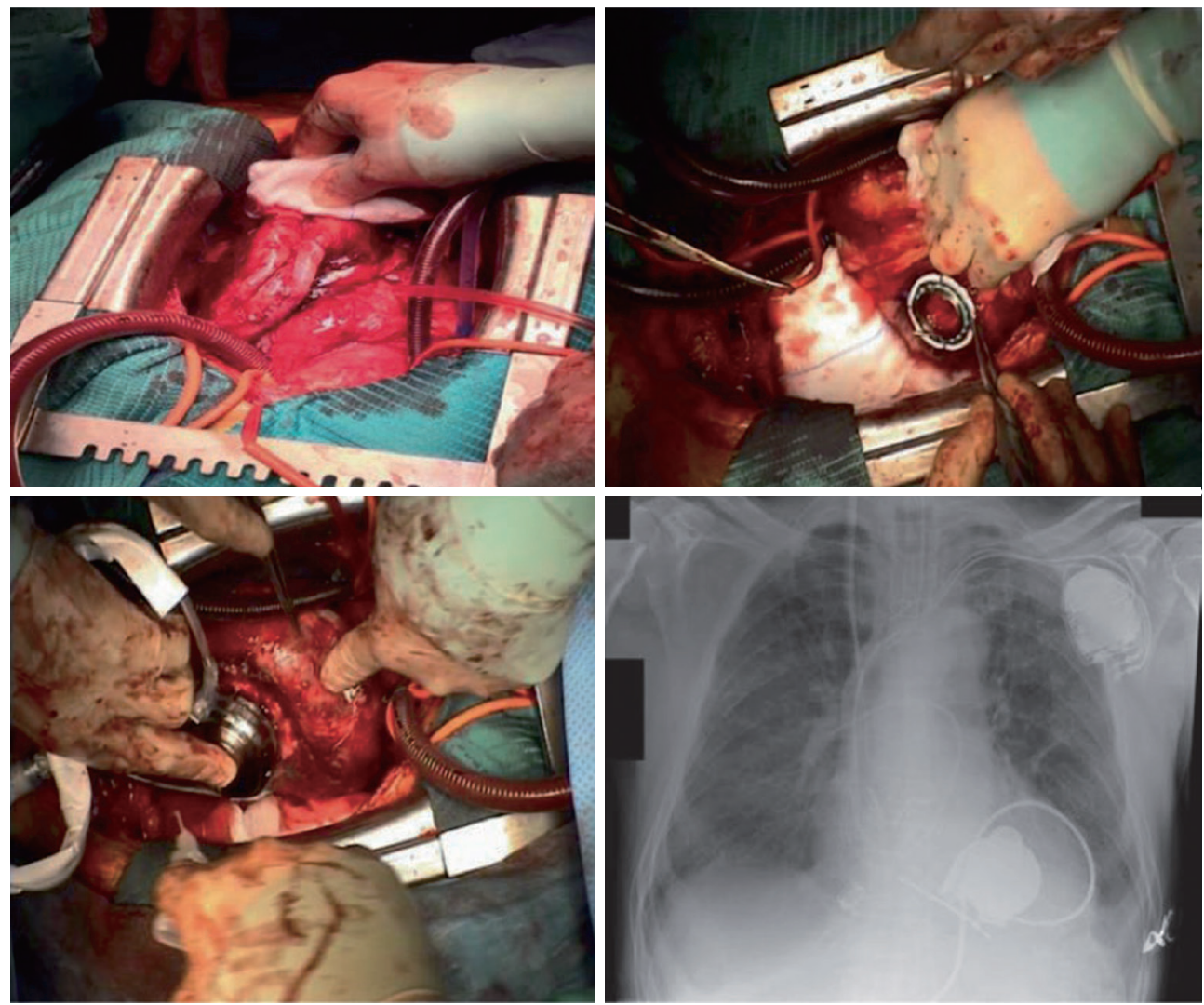

Figure 6 Posterior left ventricular assist device (LVAD) implantation in the left ventricular diaphragmatic wall due to post-infarction left ventricle aneurysm.

and restricted LV cavity (38). If residual capacity of LV and wall tissue thickness result sufficient to prevent suction, the site of previous ventriculotomy, could be suitable for the inflow cannula; alternatively, the anastomosis could be performed to the synthetic patch, although at the expense of device stability and hemostasis. In case of restricted LV and unviable walls, it could be mandatory left atrium inflow cannulation $(38,39)$.

Inferior LV wall may be considered as alternative site (5): severely dilated LV with impaired geometry, post-infarction $\mathrm{LV}$ apex aneurysm and narrowed-chest patients may benefit from inflow cannula placement through the diaphragmatic wall (Figure 6). Technically, LV apex is uplifted and sewing ring site is identified at the "Frazier's point", which is located laterally to the posterior descending coronary artery, at the first third of the distance from the apex to the base of the heart, far away from the papillary muscles (40). The correct position and the absence of cannula obstructions may be checked with epicardial ultrasound or digital exploration during CPB. After coring, inflow cannula is inserted along the short axis of the $\mathrm{LV}$, without reaching the interventricular septum. The pump is rotated with the outflow directed towards the right side of the heart, allowing outflow anastomosis to the ascending aorta (40).

\section{Outflow graft positioning}

The preferential outflow site is the ascending aorta on the right curvature at about $2 \mathrm{~cm}$ above the sino-tubular junction (Figure 2) (5). After side clamping of the aorta, anastomosis should be performed in an end-to-side fashion with a $5 / 0$ prolene running suture with $45^{\circ}$ angle. This allows to physiologically direct the flow, reducing the hemodynamic burden on the root wall and on the aortic valve and, consequently, lowering the incidence of late aortic regurgitation (41-43).

The course of the graft should be intrapericardial $(5,37)$ : during minimally invasive sternotomy-sparing approaches the graft is tunneled from left side to the ascending aorta. It must be considered that this is not always a viable option 


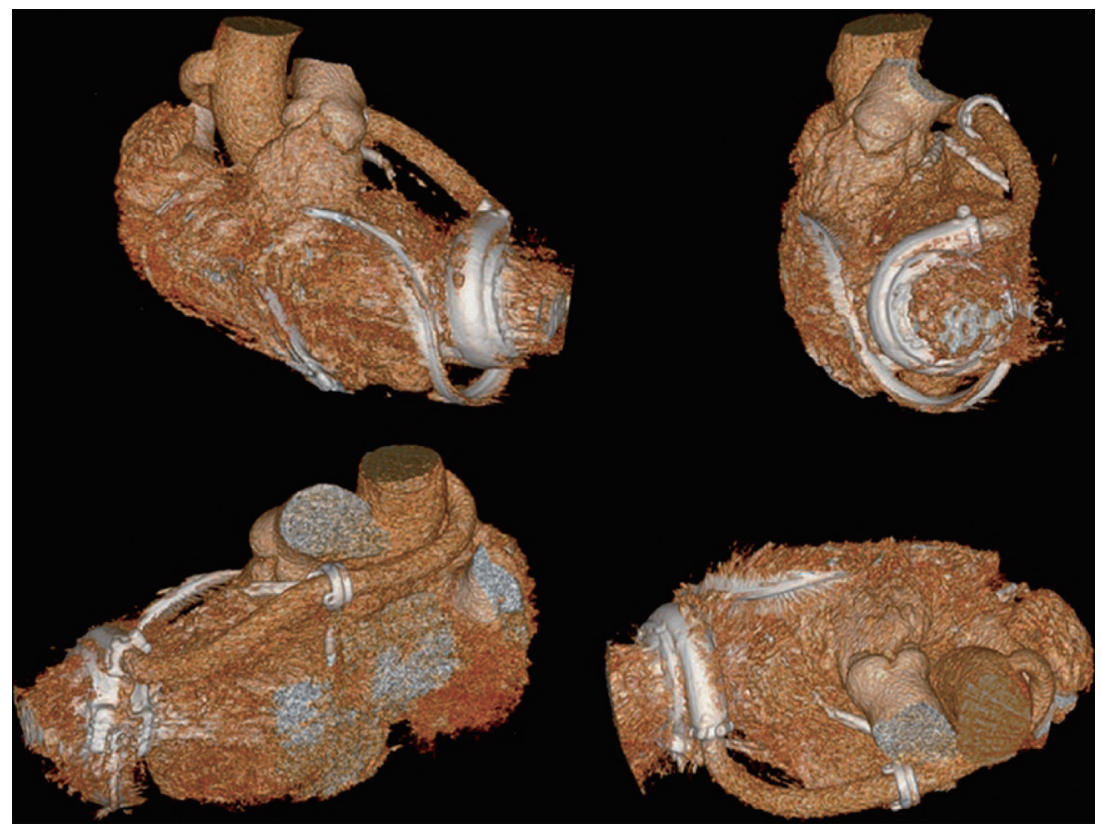

Figure 7 Outflow graft tunneling through the transverse sinus for left ventricular assist device (LVAD) implantation.

in patients with previous cardiac surgeries with strong pericardial adhesion.

Outflow line should run along the inferior RV surface and laterally to the right atrium in order to avoid crossing $\mathrm{RV}$ outflow tract. Care must be taken to avoid kinking and twisting of the outflow graft with the help of the longitudinal line markers. Graft length may be easily adjusted with clockwise or counter-clockwise rotation of the pump $(5,37)$.

An alternative course of the outflow line in minimally invasive approach and BTT strategy may be through the transverse sinus (Figure 7) $(44,45)$. This technique allows to protect LVAD graft from potential positional changes after sternal closure, and enable easier and safer re-entry during the eventual heart transplantation. Once transverse sinus has been identified and the membrane behind the aorta has been tunneled, a forcep is inserted through the sinus, from the right to the left, and outflow graft is pulled towards the standard anastomosis site on the ascending aorta. A GoreTex membrane covering the outflow is positioned in order to avoid strong adhesions. Some remarkable concerns have been reported about covering the strain relief and outflow graft with polytetrafluoroethylene (PTFE): despite its antiadherencial function, it could enhance thrombus formation between graft and PTFE itself, causing ab-extrinsic obstruction of the outflow (43).
HeartWare HVAD is the authors' choice for such a described surgical approach. However, in patients with previous cardiac surgery, this technique is not recommendable because the transverse sinus often results virtual $(44,45)$.

In case of heavy calcification, proximal graft anastomosis, abscess or pseudoaneurysms, the ascending aorta is contraindicated as outflow graft anastomosis site (5).

Alternative sites, such as descending thoracic aorta, supra-celiac abdominal aorta, innominate artery or subclavian arteries, have been described with good results (Figures 8,9) $(5,8,12,17,29,30,46-49)$.

\section{Outflow graft anastomosis to the descending aorta}

The outflow graft anastomosis to the descending aorta is integral part of "lateral implantation" previously described (Figure 8) $(5,8,12,17,29,30,46-47)$, which represents the ideal technique in case of sternal re-entry, especially in the presence of CABG. After thoracotomy, the inferior pulmonary ligament has to be divided in order to make a free pathway to the aorta. Descending aorta is exposed from pulmonary hilum to the diaphragm. CPB is then established, the coring process is performed, LVAD secured and the outflow graft is measured and deaired. The outflow is buried in the left pulmonary fissure and anastomosed 

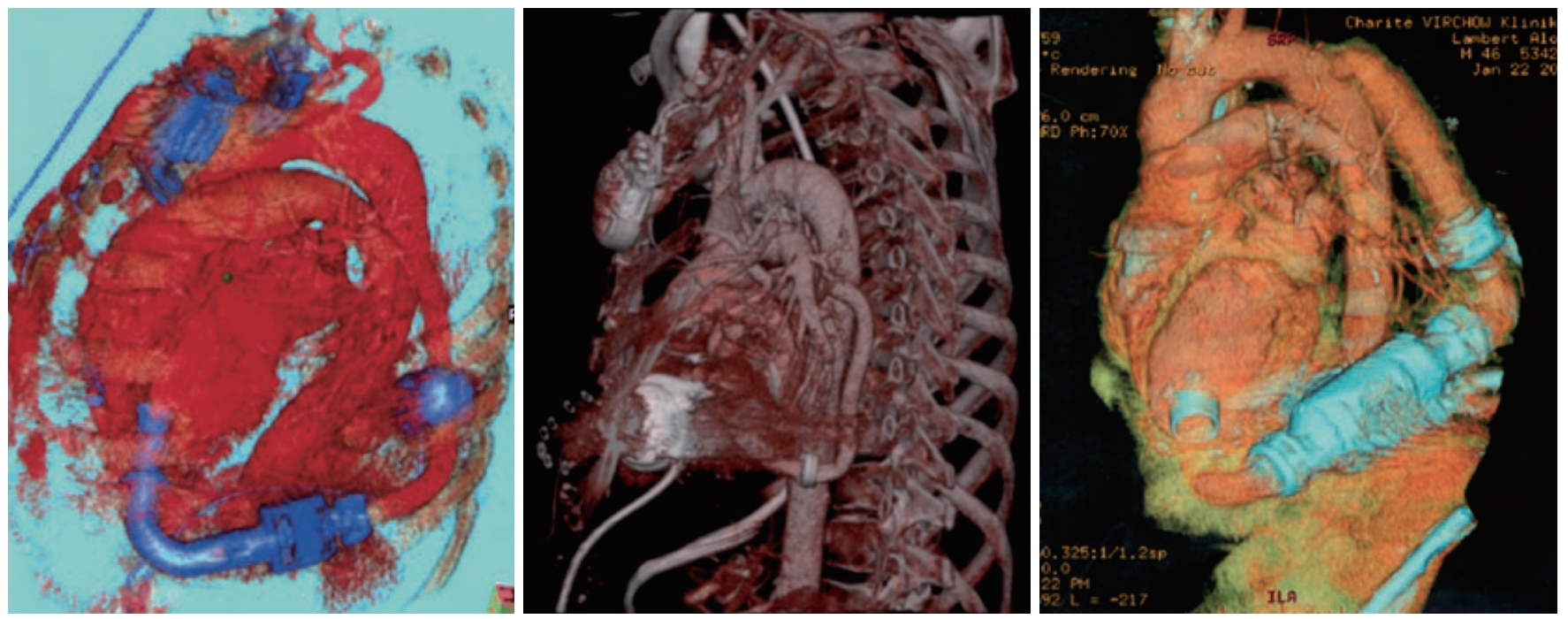

Figure 8 Outflow graft placement in the descending aorta for left ventricular assist device (LVAD) implantation.

end-to-side to the aorta during lateral partial clamping $(17,29,30,46)$. Clinical benefit due to avoided re-sternotomy have to be weighed against potential risk of a retrograde flow provided by distal anastomosis sites, that leads to "smoke" areas in the upstream aorta and aortic valve deterioration (46).

Both HeartWare HVAD and HeartMate 3 are the authors choice for such a described surgical approach.

\section{Outflow graft anastomosis to the supra-celiac abdominal aorta}

If descending aorta has calcifications or atherosclerotic plaques, the supra-celiac abdominal aorta may be used as outflow anastomosis site $(5,12,17,29,30,46)$. A left subcostal incision is preferred in this case. Aorta is exposed with an extra-peritoneal access and the left diaphragm is divided to expose the heart. After femoral CPB initiation, the LVAD is implanted on the diaphragmatic LV wall; the outflow graft is then anastomosed, as previously described (47).

HeartWare HVAD is the authors' choice for such a described surgical approach.

\section{Outflow graft anastomosis to the innominate artery}

Outflow graft anastomosis to the innominate artery is optimal in BTT strategy and is a good alternative in case of unviable ascending aorta. During standard approach

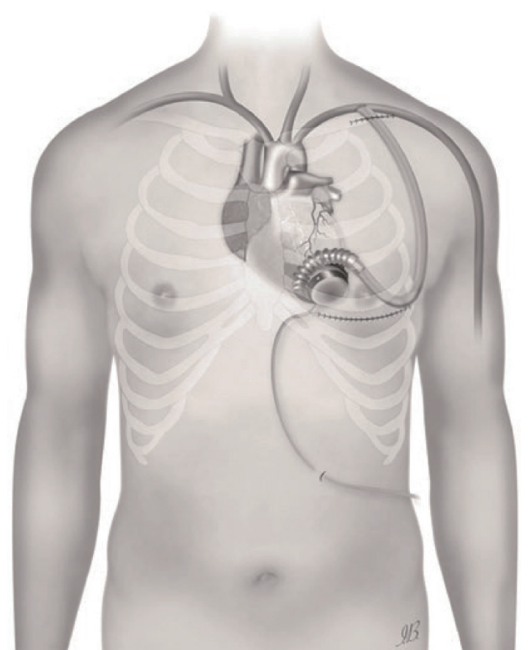

Figure 9 Axillary artery outflow graft placement in less invasive left ventricular assist device (LVAD) implantation.

with sternotomy, the outflow graft runs along with RV profile, between the pericardium and the right atrium. Then, it passes over the superior vena cava and deeply to the innominate vein to be anastomosed to the innominate artery. During minimally invasive approach (upper hemisternotomy is the preferable access), the outflow may be tunneled either intrapericardially or through the left pleural cavity in REDO cases $(5,8,12,37,47-49)$.

HeartWare HVAD is the authors' choice for such a described surgical approach. 

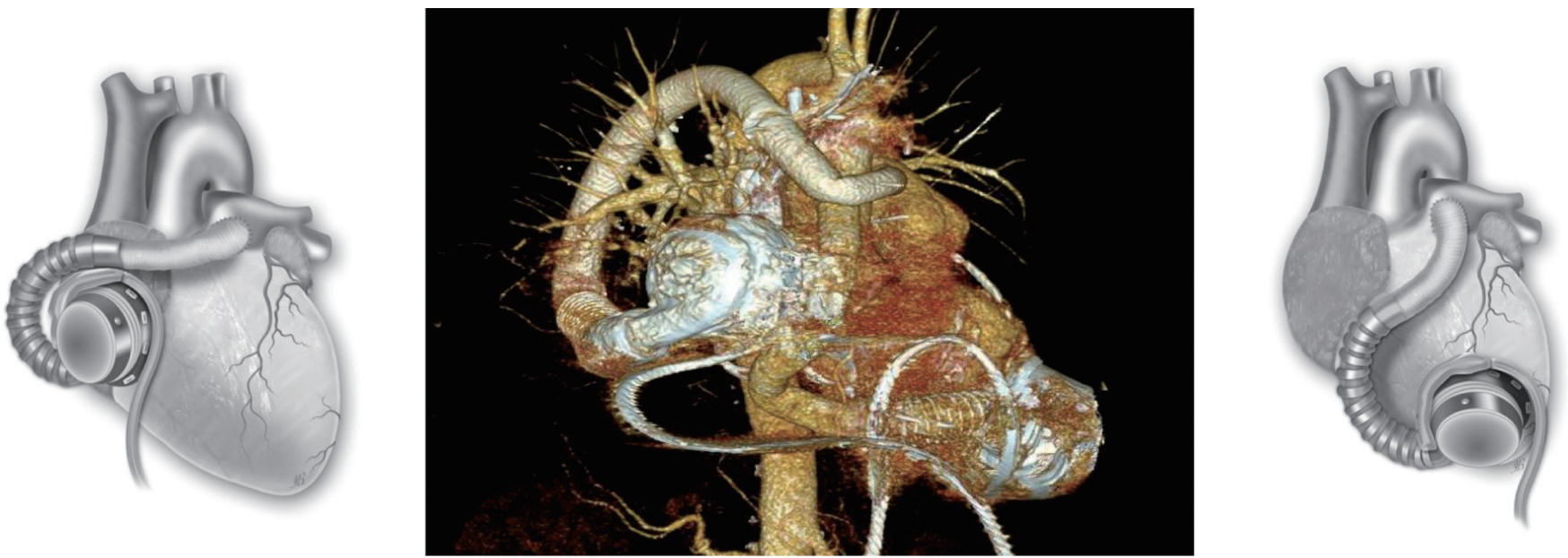

Figure 10 Biventricular implantable ventricular assist device implantation options.

\section{Outflow graft anastomosis to the axillary artery}

Both left and right axillary arteries may be considered as outflow graft anastomosis sites (Figure 9) $(5,8,12,37,46-49)$. Despite its advantage in minimally invasive setting, this choice presents three main technical issues: the small caliber of axillary artery, intra-thoracic tunnelization through an intercostal space and the risk of left arm overflow and peripheral malperfusion.

After a subclavian or a delto-pectoral incision, axillary artery is exposed: usually, the small size of vessels makes this technique compatible with devices whose outflow vascular graft has a diameter no more than $10 \mathrm{~mm}$. The anastomosis may be performed in different ways according to axillary artery diameter: if diameter is higher than $8 \mathrm{~mm}$, end-toside or end-to-end fashion (combined with side anastomosis of distal axillary) could be used. Viceversa, smaller arteries may require a $8 \mathrm{~mm}$ diameter prosthesis bridge of $2-2.5 \mathrm{~mm}$ in length and a graft-to-graft anastomosis. The graft is protected with ring-reinforced Gore-Tex membrane, preventing from kinking and compression, and tunneled at the first or second intercostal space. In order to avoid distal overflow, axillary artery bending is recommended: after pump start, bending is tightened until right and left radial pressures are balanced $(8,12,47,49)$. Right axillary artery anastomosis has been described during HeartMate II implantation in the setting of heavily calcified ascending and descending aorta: in this case, the outflow is tunneled through the right part of the diaphragm, right pleural cavity and second intercostal space (17). HeartWare HVAD is the authors' choice for such a described surgical approach.

\section{Biventricular assist device (BVAD) implantation}

After first reports on the newer small miniaturized LVAD systems, a full implantable continuous-flow biventricular support has been discussed and judged to be technically more feasible, by the scientific community (50-55). Since 2010 several mechanical circulatory support centres have been advocating a personal technique which allows the adoption of two implantable centrifugal LVADs of the type HeartWare $\operatorname{HVAD}^{\circledast}$ (Medtronic) and, currently, HeartMate 3 (Abbott) as a biventricular assist system (1,5,10,50-54).

Patients who need biventricular long-term support (up to $30 \%$ of advanced HF population) either present primarily with severe chronic biventricular failure or have suffered secondarily refractory RV failure just after LVAD implantation, thus requiring additional long-term RV mechanical support.

Patients who present a big chest may receive the insertion of the pump to the RV free wall. In small patients it is recommend insertion of the pump to the right atrium or to the diaphragmatic RV wall (Figure 10).

To provide a 'physiological' flow range of 3 to 6 liters per minute within a system speed setting of between 2,300 and 3,600 rpms, as usually set when the $\mathrm{HVAD}^{\circledR}$ is used in terms of LVAD configuration, the afterload of the right system should be somehow increased. The Berlin team recommends to reduce the outflow graft diameter to let the RVAD afterload reach the levels of a systemic circulation, artificially (50-52). This can be done by reducing the graft diameter of approximately $5 \mathrm{~mm}$ in patients with normal and 6 to $7 \mathrm{~mm}$ in patients with elevated pulmonary vascular 
resistance (5,50-52). Graft reduction can be performed by side-clamping and narrowing the graft with a prolene suture or by placement of titanium clips. An Hegar bar is adopted for calibration. The length of outflow conduit narrowing should be about $30 \mathrm{~mm}$ thus influencing the RVAD afterload according to Hagen-Poiseuille law (50-55).

To reduce the length of the inflow cannula to be inserted into the right ventricle, it is recommended to add, ideally, two $5 \mathrm{~mm}$ silicon suture rings (Berlin Heart GmbH, Berlin, Germany) to the original $\mathrm{HVAD}^{\circledR}$ implantation ring $(5,12,50,51)$. If this is not available, hand-made rings of Dacron or Teflon felt velour can be tailored and adopted. This strategy prevents the cannula from deep penetration into the RV chamber.

More than 300 CF-BVADs have been implanted worldwide $(1,10)$, even in the pediatric population (53-55). We prefer HeartWare HVAD in the case of need for implantable mechanical biventricular support (52). Thereafter, the HeartMate 3 is set and may be adopted as BVAD too, even if being more 'bulky' with encouraging success rate outcomes (55).

Surgery required for two pulsatile pumps placement in terms of biventricular long-term support leads to an extensive and traumatic operation both for pump housing location (e.g., Thoratec IVAD, Thoratec Corp., Pleasanton, CA) and blood penetrating cannulas positioning (e.g.: Berlin Heart Excor, Berlin Heart GmbH, Berlin, Germany; Thoratec BVAD, Thoratec Corp., Pleasanton, CA; Abiocor, Abiomed Inc., Danvers, MA, USA). Thus the risk of bleeding and/or infection is high.

It should be considered the advantages of an implantable continuous-flow BVAD system in terms of greater comfort and quality of life. The two systems (both HeartWare and HeartMate 3) run completely noiselessly and, even if the patients have to carry a couple of controllers and four batteries, they report a higher degree of freedom and mobility $(5,50)$.

\section{Conclusions}

During last decades, great efforts in terms of devices design evolution and surgical techniques improvement have been made. Various LIS techniques have been developed and described, thus becoming the standard approach, in expert and high volume centers as being associated with low risk of post-operative bleeding, infection, RV failure and mortality. Alternative inflow and outflow graft sites allow tailored surgical planning for each referred patient thus expanding the cohort of recipients potentially eligible for LVAD placement. BVAD implantation remains a challenge both technically and physiologically but clinical results are encouraging. The novel versatile and miniaturized third-generation centrifugal pumps (HeartWare HVAD and HeartMate 3) allow technical feasibility of all surgical approaches described above thus being currently adopted by several institutions facing with whatever clinical need or surgical choice.

\section{Acknowledgments}

This paper was partially presented in the joint session of the 27th Annual Meeting of the ISMCS 2019 and RHICS 17th Expert Forum, October 21-23, 2019, Bologna, Italy. Funding: None.

\section{Footnote}

Provenance and Peer Review: This article was commissioned by the Guest Editor (Roland Hetzer) for the series "Heart Failure in the Young and Old: Insights into Various Therapies" published in Cardiovascular Diagnosis and Therapy. The article has undergone external peer review.

Conflicts of Interest: The authors have completed the ICMJE uniform disclosure form (available at http://dx.doi. org/10.21037/cdt-20-325). The series "Heart Failure in the Young and Old: Insights into Various Therapies" was commissioned by the editorial office without any funding or sponsorship. The authors have no other conflicts of interest to declare.

Ethical Statement: The authors are accountable for all aspects of the work in ensuring that questions related to the accuracy or integrity of any part of the work are appropriately investigated and resolved.

Open Access Statement: This is an Open Access article distributed in accordance with the Creative Commons Attribution-NonCommercial-NoDerivs 4.0 International License (CC BY-NC-ND 4.0), which permits the noncommercial replication and distribution of the article with the strict proviso that no changes or edits are made and the original work is properly cited (including links to both the formal publication through the relevant DOI and the license). See: https://creativecommons.org/licenses/by-nc-nd/4.0/. 


\section{References}

1. Kirklin JK, Naftel DC, Pagani FD, et al. Seventh INTERMACS annual report: 15,000 patients and counting. J Heart Lung Transplant 2015;34:1495-504.

2. Steinberg MH. Go with the flow. N Engl J Med 2017;376:485-7.

3. Maltais S, Kilic A, Nathan S, et al. PREVENtion of HeartMate II Pump Thrombosis Through Clinical Management: The PREVENT multi-center study. J Heart Lung Transplant 2017;36:1-12.

4. Miller LW, Pagani FD, Russell SD, et al. Use of a continuous-flow device in patients awaiting heart transplantation. N Engl J Med 2007;357:885-96.

5. Potapov EV, Antonides C, Crespo-Leiro MG, et al. 2019 EACTS Expert Consensus on long-term mechanical circulatory support. Eur J Cardiothorac Surg 2019;56:230-70.

6. Turner KR. Right Ventricular Failure After Left Ventricular Assist Device Placement-The Beginning of the End or Just Another Challenge? J Cardiothorac Vasc Anesth 2019;33:1105-21.

7. Imamura T, Nguyen A, Chung B, et al. Association of Inflow Cannula Position with Left Ventricular Unloading and Clinical Outcomes in Patients with HeartMate II Left Ventricular Assist Device. ASAIO J 2019;65:331-5.

8. Maltais S, Davis ME, Haglund N. Minimally invasive and alternative approaches for long-term LVAD placement: the Vanderbilt strategy. Ann Cardiothorac Surg 2014;3:563-9.

9. Anyanwu AC. Technique for less invasive implantation of heartmate II left ventricular assist device without median sternotomy. Semin Thorac Cardiovasc Surg 2011;23:241-4.

10. Kirklin JK, Cantor R, Mohacsi P, et al. First Annual IMACS Report: A global International Society for Heart and Lung Transplantation Registry for Mechanical Circulatory Support. J Heart Lung Transplant;2016;35:407-12.

11. Papathanasiou M, Tsourelis L, Pizanis N, et al. Resternotomy does not adversely affect outcome after left ventricular assist device implantation. Eur J Med Res 2017;22:46.

12. Krabatsch T, Drews T, Potapov E, et al. Different surgical strategies for implantation of continuous-flow VADsExperience from Deutsches Herzzentrum Berlin. Ann Cardiothorac Surg 2014;3:472-4.

13. Hill JD, Avery GJ, Egrie G, et al. Less invasive Thoratec LVAD insertion: a surgical technique. Heart Surg Forum 2000;3:218-23.
14. Gregoric ID, La Francesca S, Myers T, et al. A less invasive approach to axial flow pump insertion. J Heart Lung Transplant 2008;27:423-6.

15. Gregoric ID, Bruckner BA, Jacob L, et al. Clinical Experience With Sternotomy Versus Subcostal Approach for Exchange of the HeartMate XVE to the HeartMate II Ventricular Assist Device. Ann Thorac Surg 2008;85:1646-9.

16. Samuels LE, Casanova-Ghosh E, Rodriguez R, et al. Left ventricular assist device implantation in high risk destination therapy patients: an alternative surgical approach. J Cardiothorac Surg 2012;7:21.

17. Riebandt J, Sandner S, Mahr S, et al. Minimally invasive thoratec Heartmate II implantation in the setting of severe thoracic aortic calcification. Ann Thorac Surg 2013;96:1094-6.

18. Cheung A, Lamarche Y, Kaan A, et al. Off-Pump Implantation of the HeartWare HVAD Left Ventricular Assist Device Through Minimally Invasive Incisions. Ann Thorac Surg 2011;91:1294-6.

19. Popov AF, Hosseini MT, Zych B, et al. HeartWare Left Ventricular Assist Device Implantation Through Bilateral Anterior Thoracotomy. Ann Thorac Surg 2012;93:674-6.

20. Deniz E, Chatterjee A, Feldmann C, et al. How to do it: Tips and tricks of minimal-invasive HVAD® implantationThe lateral approach. J Thorac Dis 2018;10:S1829-33.

21. Schmitto JD, Molitoris U, Haverich A, et al. Implantation of a centrifugal pump as a left ventricular assist device through a novel, minimized approach: Upper hemisternotomy combined with anterolateral thoracotomy. J Thorac Cardiovasc Surg 2012;143:511-3.

22. Haberl T, Riebandt J, Mahr S, et al. Viennese approach to minimize the invasiveness of ventricular assist device implantation. Eur J Cardiothorac Surg 2014;46:991-6.

23. McGee E, Danter M, Strueber M, et al. Evaluation of a lateral thoracotomy implant approach for a centrifugalflow left ventricular assist device: The LATERAL clinical trial. J Heart Lung Transplant 2019;38:344-51.

24. Gosev I, Wood K, Ayers B, et al. Implantation of a fully magnetically levitated left ventricular assist device using a sternal-sparing surgical technique. J Heart Lung Transplant 2020;39:37-44.

25. Schmitto JD, Krabatsch T, Damme L, et al. Less invasive HeartMate 3 left ventricular assist device implantation. J Thorac Dis 2018;10:S1692-5.

26. Riebandt J, Wiedemann D, Laufer G, et al. Sternotomy Sparing Thoratec HeartMate 3 Implantation Via Bilateral Minithoracotomy. Innovations (Phila) 2018;13:74-6. 
27. Pasrija C, Sawan MA, Sorensen E, et al. Less invasive left ventricular assist device implantation may reduce right ventricular failure. Interact Cardiovasc Thorac Surg 2019;29:592-8.

28. Wert L, Chatterjee A, Dogan G, et al. Minimally invasive surgery improves outcome of left ventricular assist device surgery in cardiogenic shock. J Thorac Dis 2018;10:S1696-702.

29. Maltais S, Anwer LA, Tchantchaleishvili V, et al. Left lateral thoracotomy for centrifugal continuous-flow left ventricular assist device placement: An analysis from the mechanical circulatory support research network. ASAIO J 2018;64:715-20.

30. Umakanthan R, Haglund NA, Stulak JM, et al. Left thoracotomy heartware implantation with outflow graft anastomosis to the descending aorta: A simplified bridge for patients with multiple previous sternotomies. ASAIO J 2013;59:664-7.

31. Dell'Italia LJ. Anatomy and physiology of the right ventricle. Cardiol Clin 2012;30:167-87.

32. Khalpey Z, Riaz IB, Marsh KM, et al. Robotic left ventricular assist device implantation using left thoracotomy approach in patients with previous sternotomies. ASAIO J 2015;61:e44-6.

33. Khalpey Z, Sydow N, Slepian MJ, Poston R. How to do it: Thoracoscopic left ventricular assist device implantation using robot assistance. J Thorac Cardiovasc Surg 2014;147:1423-5.

34. Chivukula VK, Beckman JA, Prisco AR, et al. Left Ventricular Assist Device Inflow Cannula Angle and Thrombosis Risk. Circ Heart Fail 2018;11:e004325.

35. Chivukula VK, Beckman JA, Prisco AR, et al. Small Left Ventricular Size Is an Independent Risk Factor for Ventricular Assist Device Thrombosis. ASAIO J 2019;65:152-9.

36. Kawabori M, Kurihara C, Sugiura T, et al. ContinuousFlow Left Ventricular Assist Device Implantation in Patients With a Small Left Ventricle. Ann Thorac Surg 2018;105:799-806.

37. Slaughter MS. Implantation of the heartware left ventricular assist device. Semin Thorac Cardiovasc Surg 2011;23:245-7.

38. Osaki S, Edwards NM, Kohmoto T. Strategies for Left Ventricular Assist Device Insertion After the Dor Procedure. J Heart Lung Transplant 2009;28:520-2.

39. Kervan U, Unal EU, Sert DE, et al. Heartware left ventricular assist device implantation combined with surgical ventricular reconstruction. Exp Clin Transplant
2019;17:278-80.

40. Gregoric ID, Cohn WE, Frazier OH. Diaphragmatic implantation of the HeartWare ventricular assist device. J Heart Lung Transplant 2011;30:467-70.

41. Bouabdallaoui N, El-Hamamsy I, Pham M, et al. Aortic regurgitation in patients with a left ventricular assist device: A contemporary review. J Heart Lung Transplant 2018;37:1289-97.

42. May-Newman K, Hillen B, Dembitsky W. Effect of left ventricular assist device outflow conduit anastomosis location on flow patterns in the native aorta. ASAIO J 2006;52:132-9.

43. Alnabelsi T, Shafii AE, Gurley JC, et al. Left ventricular assist device outflow graft obstruction: A complication specific to polytetrafluoroethylene covering. A word of caution! ASAIO J 2019;65:e58-62.

44. Hanke JS, Rojas SV, Cvitkovic T, et al. First results of HeartWare left ventricular assist device implantation with tunnelling of the outflow graft through the transverse sinus. Interact Cardiovasc Thorac Surg 2017;25:503-8.

45. Loforte A, Gliozzi G, Coppola G, et al. Outflow graft tunneling through the transverse sinus for HeartWare HVAD implantation as bridge to heart transplantation strategy. Multimed Man Cardiothorac Surg 2019. doi: 10.1510/mmcts.2019.012.

46. Kar B, Delgado RM, Frazier OH, et al. The effect of LVAD aortic outflow-graft placement on hemodynamics and flow: Implantation technique and computer flow modeling. Tex Heart Inst J 2005;32:294-8.

47. El-Sayed Ahmed MM, Aftab M, Singh SK, et al. Left ventricular assist device outflow graft: alternative sites. Ann Cardiothorac Surg 2014;3:541-5.

48. Hanke JS, Rojas SV, Martens A, et al. Minimally invasive left ventricular assist device implantation with outflow graft anastomosis to the innominate artery. J Thorac Cardiovasc Surg 2015;149:e69-70.

49. Carrozzini M, Bejko J, Guariento A, et al. Minimally Invasive implanatation of continuous Flow Left Ventricular Assist Devices: the evolution of surgical techniques in a single-center experience. Artif Organs 2019;43:E41-52.

50. Hetzer R, Krabatsch T, Stepanenko A, et al. Long-term biventricular support with the heartware implantable continuous flow pump. J Heart Lung Transplant 2010;29:822-4.

51. Krabatsch T, Stepanenko A, Schweiger M, et al. Alternative technique for implantation of biventricular support with heartware implantable continuous flow pump. ASAIO J 2011;57:333-5. 
52. Loforte A, Montalto A, Della Monica PL, et al. Biventricular support with the HeartWare implantable continuous flow pump: an additionale contribution. J Heart Lung Transplant 2010;29:1443-4.

53. Stein ML, Yeh J, Reinhartz O, et al. HeartWare HVAD for biventricular support in children and adolescents: The Stanford Experience. ASAIO J 2016;62:e46-51.

Cite this article as: Loforte A, Gliozzi G, Mariani C, Cavalli GG, Martin-Suarez S, Pacini D. Ventricular assist devices implantation: surgical assessment and technical strategies. Cardiovasc Diagn Ther 2021;11(1):277-291. doi: 10.21037/cdt20-325
54. Schweiger M, Krüger B, Cavigelli-Brunner A, et al. Biventricular intracorporeal ventricular assist device in a 10-year-old child. Int J Artif Org 2015;38:233-5.

55. Lavee J, Mulzer J, Krabatsch T, et al. An international multicenter experience of biventricular support with HeartMate 3 ventricular assist systems. J Heart Lung Transplant 2018;37:1399-402. 